\title{
Estudio de la variación de la permeabilidad de la dentina radicular usando isótopos 99m Tc, después de la aplicación de láser de alta intensidad
}

\section{Study of the dentin root permeability variation using ${ }^{99 m} T c$ isotopes, after the application of high intensity laser}

\section{Cabrales Salgado R*, Yamazaki-Arasaki A**, Mónica Kleine B***, Prokopowitsch I****}

\section{RESUMEN}

Objetivo: Este estudio tuvo como objetivo evaluar las variaciones de la permeabilidad dentinaria radicular de dientes humanos después del preparo químico-quirúrgico e irradiación con láser de Er: YAG, Nd: YAG, a través de la utilización del radioisótopo tecnecio-99m $\left({ }^{99 m} \mathrm{Tc}\right)$ libre.

Materiales y métodos: Canales de treinta raíces palatinas de primeros premolares superiores humanos fueron preparados y divididos, aleatoriamente, en 3 grupos experimentales: G1 (control), G2 (irradiación con láser de Er: YAG) y G3 (irradiación con láser de Nd: YAG). Las muestras recibieron en el interior del canal radicular, ${ }^{99 \mathrm{~m} T c}$ libre en cantidad suficiente para el llenado y colocados en tubos de ensayo contiendo $1 \mathrm{ml}$ de solución salina. Después de 1 hora, la cuenta del isótopo radioactivo $\left({ }^{99 \mathrm{~m} T c)}\right.$ fue realizada.

Resultados: Después del análisis estadístico (ANOVA), fue verificado que los mejores resultados obtenidos en cuanto a la disminución de la permeabilidad se encontraron en el Grupo 3, seguido del Grupo 2 y del Grupo 1. Conclusión: Concluimos que irradiación con láser de Nd: YAG provocó una disminución significativa de la permeabilidad dentinaria del canal radicular. El láser de Er: YAG y el control en relación a la permeabilidad de la dentina de las paredes del canal radicular no presentaron diferencias estadísticamente significativas a nivel de $5 \%$.

Palabras clave: Endodoncia, permeabilidad de la dentina radicular, Er:YAG, Nd:YAG, radioisótopo tecnecio99m (DeCS Bireme).

\section{SUMMARY}

Objective: The objective of this study was to evaluate changes in root dentin permeability of human teeth after chemical-surgical preparation, and laser irradiation with Er: YAG, Nd: YAG, through the use of the radioisotope technetium-99m ( ${ }^{99 m}$ Tc) free.

* DDS, Ph.D. Endodoncia. Universidad de São Paulo Brasil. Profesor invitado Facultad de odontología. Universidad de Cartagena. Colombia.

** DDS, Ms, Ph.D. Endodoncia. Universidad de São Paulo Brasil. Profesora Universidad Cruzeiro do Sul. São Paulo. Brasil.

*** DDS. Especialista en Endodoncia. Estudiante de maestría en Ciencias odontológicas con área de concentración en endodoncia. Universidad de São Paulo Brasil. Universidad Cruzeiro do Sul. São Paulo. Brasil.

**** DDS, Ms, Ph.D. Endodoncia. Universidad de São Paulo Brasil. Profesor Titular Universidad Cruzeiro do Sul. São Paulo. Brasil. 
Materials and methods: thirty Channels palatal roots of human maxillary first premolars were prepared and divided randomly into three experimental groups: G1 (control), G2 (laser irradiation with Er: YAG) and G3 (Nd laser irradiation : YAG). in the samples were deposited ${ }^{99 \mathrm{~m}} \mathrm{Tc}$ free inside the root canal, enough to fill and placed in test tubes containing $1 \mathrm{ml}$ of saline. After 1 hour, the account of the radioactive isotope $\left({ }^{99 \mathrm{~m} T c)}\right.$ ) was performed. Results: After statistical analysis (ANOVA) found that the best results in terms of decreased permeability were found in Group 3, followed by Group 2 and Group 1.

Conclusion: We conclude that irradiation with Nd: YAG caused a significant decrease in the permeability of root canal dentin. Laser Er: YAG and control in relation to the dentin permeability of root canal walls showed no statistically significant differences at $5 \%$.

Key words: Endodontic, root dentin permeability, Er: YAG, Nd: YAG, radioisotope technetium-99m (MeSH Database).

Fecha de recepción: Noviembre 2010.

Aceptado para publicación: Julio 2011.

Cabrales Salgado R, Yamazaki-Arasaki A, Mónica Kleine B, Prokopowitsch I. Estudio de la variación de la permeabilidad de la dentina radicular usando isótopos ${ }^{99 \mathrm{~m}} \mathrm{Tc}$, después de la aplicación de láser de alta intensidad. Av. Odontoestomatol 2012; 28 (1): 39-46.

\section{INTRODUCCIÓN}

La permeabilidad es una característica del tejido dentinario, es decir, la capacidad que la dentina tiene de promover la difusión de sustancias para su interior. Esta característica permeable de la dentina se debe principalmente a la presencia de innumerables túbulos, que inciden con más prevalencia en áreas próxima a la cámara pulpar y con menor incidencia en el tercio apical $(1,2)$.

A finales del siglo pasado el aumento de la permeabilidad dentinaria radicular ha sido una condición indispensable para la limpieza y desinfección de los dientes tratados endodónticamente. Por esta razón, innumerables son los métodos empleados para el análisis del acondicionamiento de las paredes dentinarias y del sistema de canales radiculares.

Entre los métodos más utilizados para identificar el índice de permeabilidad, encontramos la utilización de colorantes tales como nanquim, azul de metileno, rodamina $B$, infiltración de líquidos y utilización de radioisótopos (3-9).

Los isótopos radiactivos son formas radioactivas de elementos químicos creados mediante reacciones nucleares y utilizadas principalmente para diagnósti- co en medicina como también para evaluaciones de permeabilidad dentinaria radicular en odontología. Entre los radioisótopos sintéticos encontramos el tecnecio-99m ( $\left.{ }^{99 m} \mathrm{Tc}\right)$, que se obtiene por desintegración beta del molibdeno $\left({ }^{99 \mathrm{~m} M o}\right)$ y es el radiofármaco más utilizado para el empleo clínico cintilográfico en la medicina nuclear, ya que su decaimiento de radiactividad es de 6,02 horas $(9,10)$.

Los láseres de Nd: YAG y Er: YAG han sido reportados en la literatura como coadyuvantes en la limpieza y desinfección de canales radiculares, mostrando su eficacia en la remoción del smear layer, además, provocan alteraciones estructurales que influyen directamente en la permeabilidad dentinaria $(11,18)$.

Diversos estudios reportan que la irradiación con láser de Nd: YAG provoca derretimiento y fusión del tejido dentinario, obliterando así la entrada de los canalículos, causando una reducción de su permeabilidad, mientras que la irradiación con Er: YAG causa erosión y rugosidades en la superficie dentinaria; la abertura y la eliminación de smear layer en los túbulos dentinarios y por consecuencia un incremento de la permeabilidad de la dentina (22-24).

Esos resultados podrían explicarse por la diferencia de las longitudes de onda (Er: YAG y $\lambda=2,94 \mathrm{mi}-$ 
cras, Nd: YAG $=1,06 \mu \mathrm{m})$ y por la diferencia en la interacción y afinidad de los láseres por los compuestos presentes en el tejido dentinario.

El éxito parcial de las conductas antimicrobianas del tratamiento endodóntico, proponen la irradiación con Láser, como una terapéutica ideal a ser asociada para la eliminación bacteriana y alteraciones de permeabilidad dentinaria radicular. Las investigaciones experimentales y clínicas destacan las propiedades antimicrobianas y las alteraciones estructurales que provocan variaciones de permeabilidad dentinaria radicular que inducen los láseres de lata potencia de Er:YAG, y Nd:YAG entre las longitudes de onda estandarizadas para aplicaciones en la endodoncia por diversos autores como Takeda, Biedma, Pécora, Aranha entre otros $(13,22-27)$. Señalando que las propiedades de la luz Láser de alta potencia en contacto con estructura superficial de la dentina radicular ocasiona una alteración morfológica por ablación o fusión y recristalización del área irradiada. Por estos motivos se hace necesario evaluar alteraciones de la permeabilidad dentinaria radicular con una metodología más precisa como es la utilización de marcadores radioactivos, después de irradiadas las superficies dentinarias radiculares con láseres de alta potencia de Er:YAG, y Nd:YAG.

El objetivo de este estudio fue evaluar los índices de permeabilidad de la dentina radicular, usando el método cuantitativo de la penetración de radioisótopo ${ }^{99 m}$ Tc después del preparo químico-quirúrgica del canal radicular, con o sin irradiación con láser de Er: YAG y Nd: YAG, respectivamente.

\section{MATERIALES Y MÉTODOS}

Para la realización del presente estudio experimental in Vitro con fines terapéuticos.

El proyecto de investigación fue aprobado por el Comité de Ética en investigación de la Facultad de Odontología da Universidad de São Paulo, bajo el parecer de aprobación, protocolo 137/07.

Fueron seleccionados, de la Clínica Odontológica del curso de especialización de Cirugía y Traumatología Buco maxilofacial de la FUNDECTO, pacientes con edades entre 12 y 20 años de edad, donadores (conforme Termino de Consentimiento Libre Esclarecido) de 30 primeros premolares superiores humanos hígidos, dotados de completa formación radicular con raíces separadas, indicados para exodoncia por razones ortodónticas.

Durante la realización de procesos endodónticos, fue realizada la cirugía de acceso a la cámara pulpar con puntas esféricas diamantadas 1016HL, 1012HL (proporcional al volumen de la cámara pulpar), refrigeradas, complementando con brocas Endo Z, para promover la divergencia de las paredes axiales de la cámara pulpar.

Con pieza de baja velocidad, se usan fresas GatesGlidden y Largo para el preparo de la entrada al canal, siempre irrigando profusamente la cámara pulpar y la entrada de los canales con hipoclorito de sodio al $1 \%$, concomitantemente con aspiración.

En el canal lingual fue realizada la pulpectomía, con la lima tipo K \#10 irrigando con hipoclorito de sodio al $1 \%$, con el propósito de eliminar cualquier material presente en el interior del canal. La penetración del instrumento fue realizó hasta llegar a la longitud real del conducto.

La longitud real de trabajo fue determinada restando $1 \mathrm{~mm}$ de la medida obtenida a partir de la introducción de la lima tipo K \#15 en el canal radicular hasta el foramen apical (longitud real de la canal), observada con la ayuda de una lupa $8 x$.

Durante la instrumentación manual, fue utilizada crema RC-PREP e hipoclorito de sodio al $1 \%$, verificando la reacción de efervescencia entre los productos químicos.

La instrumentación fue realizó con limas manuales tipo K y la primera lima que fue escogida fue aquella que ajustó en la longitud de trabajo, seguida por cuatro instrumentos más, y este último se utilizó para la preparación apical. Finalizando el preparo apical se realizó la limpieza final del canal radicular con irrigación y aspiración de $10 \mathrm{ml}$ de hipoclorito de sodio al $1 \%$, seguido de $10 \mathrm{ml}$ de EDTA-T al $17 \%$ y la instrumentación manual con la lima de preparación apical. 
Con la ayuda de un disco diamantado adaptado a un mandril y contra-ángulo de la pieza de baja velocidad, se individualizó la raíz lingual, previamente estandarización de la longitud de las raíces en $10 \mathrm{~mm}$ de apical a cervical.

Después de secar los canales con conos de papel, los especímenes fueron divididos aleatoriamente en tres grupos experimentales con 10 raíces en cada uno.

Para la dignación aleatoria se estableció un muestreo probabilístico con el método aleatorio simple, a partir de una lista completa de la muestra, en la cual se asigno un código a cada individuo y finalmente, mediante de una tabla de números aleatorios en Excel se seleccionaron los especímenes que hicieron parte de cada grupo.

Grupo 1 (G-1: $n=10)$ de control, en el grupo 2 (G2: $n=10)$, los canales sufrieron 4 irradiaciones con Er: YAG (2.940 nm - KaVo KEY II - Kavo Co, Alemania), realizando un movimiento helicoidal; ápico-cervical $0,5 \mathrm{~s} / \mathrm{mm}$, con un intervalo de 20 segundos entre las aplicaciones, utilizando fibra de $375 \mu \mathrm{m}$ de diámetro, dentro de los parámetros establecidos en $100 \mathrm{~mJ}, 10 \mathrm{~Hz}, 1 \mathrm{~W}$, en el grupo 3 (G-3: $n=10$ ) los canales sufrieron 4 irradiaciones con láser de $\mathrm{Nd}$ : YAG (1064 nm, ADT, EE.UU.) realizando un movimiento helicoidal; ápico-cervical $0,5 \mathrm{~s} / \mathrm{mm}$ con un intervalo de 20 segundos entre las aplicaciones, utilizando fibra de $300 \mu \mathrm{m}$ de diámetro, dentro de los parámetros establecidos en 100 mJ, $1.5 \mathrm{~W}, 15 \mathrm{~Hz}$.

\section{Evaluación de la permeabilidad de la dentina con el radioisótopo tecnecio-99m $\left({ }^{99 \mathrm{~m}} \mathrm{Tc}\right)$}

Después del período de hidratación, los especímenes de los tres grupos fueron retirados de sus frascos y secados externamente con papel absorbente. Posteriormente, los forámenes apicales das las raíces de los diferentes grupos fueron impermeabilizadas exteriormente con resina epóxica (Araldite ${ }^{\circledR}$ ) en la extensión de $1 \mathrm{~mm}^{2}$ y manteniéndose a temperatura ambiente durante 24 horas para su polimerización.

Para el uso de radiofármacos de tecnecio-99m (IPEN generador-TEC, IPEN-CNEN), en completa reduc- ción, fue necesaria la dilución en aproximadamente $0,5 \mu$ ci de agua destilada. Es importante destacar que este fármaco se envasa en frascos de vidrio herméticamente cerrados; para efectuar su transporte, es obligatorio el uso de un blindaje de plomo portátil y su manipulación se realiza también en un área blindada con plomo.

Fueron especialmente fabricadas barras metálicas (alambre CrNi, ø 0,06 mm, Dental Morelli Ltda., Brasil) que fueron fijadas en el tercio cervical de cada muestra con la ayuda de resina epoxi, en una extensión de $2 \mathrm{~mm}^{2}$ y mantenidas a temperatura ambiente durante 24 horas para su polimerización. Después, las muestras fueron sumergidas en frascos de vidrio conteniendo solución salina para la rehidratación.

Las muestras preparadas recibieron, en el interior del conducto radicular, con la ayuda de una microjeringa que contenía ${ }^{99 \mathrm{~m}} \mathrm{Tc}$ libre en cantidad suficiente para el llenado el mismo. Asegurado por las barras metálicas, las 30 muestras ( $n=10$ por grupo) fueron colocadas en tubos de ensayo que contiene $10 \mathrm{ml}$ de solución salina en cantidad suficiente para sumergir a $1 \mathrm{~mm}$ antes del borde cervical de cada raíz.

Después de 1 hora, cada muestra fue transferida a un nuevo tubo de ensayo conteniendo una nueva solución salina y cerrada con una película de PVC. El tubo de experimentación con solución salina se depositó en otro tubo para realizar el conteo del radioisótopo ${ }^{99 m} \mathrm{Tc}$. Se empleó para ello, un contador automático modelo D5002 (Cobra II, autogamma, Packard, Canberra, EE.UU.) en el que se hizo un análisis cuantitativo de la cantidad de radioisótopo libre en la solución salina. Los valores numéricos dados por el sistema de cuantificación de la radiación para la cantidad de radioisótopo encontrado en la parte intracanal y extrarradicular, fueron sumados y el equivalente correspondió al $100 \%$ de la cantidad de radiación encontrada, posteriormente, fue asignado el porcentaje correspondiente a los valores intracanales y extrarradiculares los cuales fueron transformados a porcentajes y a continuación tabulados para el proceso estadístico por medio del análisis de varianza de ANOVA y la prueba de Tukey. 
Después de esta evaluación, las muestras se sumergieron en agua destilada durante 15 días, tiempo suficiente para la completa eliminación del radioisótopo. Después de este período, las muestras fueron retiradas del agua y secadas externamente con papel absorbente y los canales con conos de papel estéril (Dentsply, Petrópolis, Brasil).

\section{RESULTADOS}

Los resultados de este estudio fueron obtenidos a partir del análisis de las muestras, para la evaluación de la permeabilidad radicular con radioisótopo ${ }^{99 \mathrm{~m}} \mathrm{Tc}$. Los valores medios de los diferentes grupos de tecnecio-99 libre, están expresados en porcentaje (\%).

Así, los mejores resultados obtenidos en cuanto a la disminución de la permeabilidad se encontraron en el Grupo 3 con 20,07\% extrarradicular y 79,93\% intrarradicular, seguido del Grupo 2 con 28,24\% extrarradicular y $71,76 \%$ intrarradicular y del Grupo 1 con $33,42 \%$ extrarradicular y $66,58 \%$ intrarradicular.

El análisis de los resultados demostró que no hubo diferencia estadísticamente significativa entre el grupo control y el grupo irradiado con Er:YAG. Se encontró diferencia estadísticamente significativa del $5 \%$ al comparar el grupo irradiado con láser de Nd:YAG al grupo irradiado con láser de Er:YAG y al grupo control, los cuales presentaron mayor permeabilidad en la dentina radicular.

\section{DISCUSION}

El éxito del tratamiento endodóntico depende de la excelencia en la realización de todas las etapas pertinentes en el plan de tratamiento, hasta la restauración definitiva y preservación del diente tratado. La eliminación de los microorganismos presentes en el sistema de conductos radiculares y la desinfección obtenida durante el tratamiento, constituyen los objetivos primordiales de la terapia endodóntica.

El uso de irradiación con láser de alta intensidad en endodoncia, cuando se aplica como coadyuvante al saneamiento de los canales radiculares, ocupa un lugar destacado, siendo de gran interés al tratamien- to endodóntico en cuanto a complementación de la desinfección y limpieza obtenida durante la preparación del canal $(11,12)$.

La irradiación con láser de alta intensidad, cuando es aplicada a la dentina radicular, produce un efecto térmico con alta capacidad bactericida, promoviendo también, a través de la acción de la energía aplicada, alteraciones estructurales de la dentina como: fusión y recristalización de la matriz de dentina o una superficie rugosa con túbulos dentinarios abiertos y libre de smear layer, pudiendo modificar la permeabilidad de la dentina radicular, alterando los índices de penetración de medicamentos en los túbulos dentinarios. Además, el tratamiento de irradiación con láser podría prevenir o mitigar la contaminación del sistema de conductos radiculares después de su irradiación $(18,19,22-24)$.

Muchos estudios sobre la permeabilidad dentinaria del canal radicular recurrieron a diferentes reveladores, tales como, isótopos radiactivos: $\mathrm{S}^{35}, \mathrm{Na}^{22}, \mathrm{I}^{131}$, $\mathrm{P}^{32}$, usados como marcadores en estudios in Vivo e in Vitro $(8,9,10)$.

Por lo tanto, evaluamos las posibles variaciones del índice de permeabilidad de la dentina radicular en raíces humanas, previamente sometida a la preparación químico-quirúrgica y posteriormente irradiadas o no con láser de Er:YAG y de Nd:YAG.

En nuestros resultados cuantitativos, para la evaluación de la permeabilidad de dentina radicular con el radioisótopo tecnecio-99m libre y analizando las medias y porcentajes de la penetración en los diferentes grupos, observamos los mejores resultados, con respecto a la disminución de la permeabilidad de la dentina radicular y se encontró que en el Grupo 3 (Nd: YAG, 20,07\% 99mTc extrarradicular y 79,93\% 99mTc intrarradicular) seguido del Grupo 2 (Er: YAG, $28,24 \%{ }^{99 m}$ Tc extrarradicular y $71,76 \%{ }^{99 m} \mathrm{Tc}$ intrarradicular) y del Grupo 1 (Control: 33,42\% 99mTc extrarradicular y $66,58 \%{ }^{99 \mathrm{~m}} \mathrm{Tc}$ intrarradicular).

Estadísticamente, a través del análisis de varianza ANOVA, con respecto a la permeabilidad de la dentina radicular, no hubo diferencia significativa del $5 \%$ ( $p>0,05)$, entre el Grupo 1 ( ${ }^{99 m} \mathrm{Tc}$ extrarradicular, $33,42 \%$ y ${ }^{99 m}$ Tc intrarradicular, 66,58\%) y el Grupo 2 
${ }^{99 \mathrm{~m}} \mathrm{Tc}$ extrarradicular, $28,24 \%$ y ${ }^{99 \mathrm{~m}} \mathrm{Tc}$ intrarradicular, $71,76 \%)$. Interesante notar que el Grupo 2 el cual recibió irradiación con Er:YAG, no aumento de forma significativa la permeabilidad de la dentina radicular, con 95\% de confianza, cuando fue comparada la permeabilidad del Grupo 1 (control), que no recibió irradiación con láser.

Estos resultados difieren de los trabajos de diversos autores que observaron un aumento en la permeabilidad cuando es irradiado con Er:YAG (202). Además, Pécora et al (25) observaron que el láser de Er:YAG, irradiado con agua destilada en el interior del canal, promueve un mayor aumento de la permeabilidad dentinaria que cuando es irradiado con $\mathrm{NaCl}$ al $1 \%$. La diferencia de los resultados puede estar relacionada con el hecho de que en nuestro experimento, los canales han sido secados antes de ser irradiados con láser de Er:YAG.

El G3-99m extrarradicular, 20,07\% e 99mTc intrarradicular, 79,93\%, presentó diferencia estadísticamente significativa de $5 \%(p>0,05)$, en su permeabilidad, cuando su media fue comparada con las medias del G1 y el G2, los cuales presentaron mayor permeabilidad dentinaria para el medio de solución salina extrarradicular. Este hecho es consistente con muchos estudios en la literatura $(18,19)$. Además, Lee et al (21), irradiaron con Nd:YAG, la superficie dentinaria impregnada con nanquim, encontraron que este láser puede ser empleado para reducir la permeabilidad dentinaria, Aranha et al (26) observaron reducción de la permeabilidad dentinaria en $26,05 \%$ cuando irradiaron con láser de Er:YAG y reducción de 19,03\% cuando irradiaron con láser de Nd:YAG.

El uso de láseres de alta potencia en endodoncia ha aumentado significativamente en las últimas décadas. Estudios han investigado las aplicaciones y beneficios de la combinación de diversos láseres en el tratamiento endodóntico, analizando la acción de esta tecnología como complemento de un tratamiento exitoso. En la actualidad se destacan, en la practica clínica los láseres de Nd: YAG, Er: YAG, Er;Cr:YSGG y diodos (20).

En este punto, vale la pena decir; que siempre en investigaciones in Vitro, se ha preocupado en aproxi- mar el experimento a condiciones reales para adquirir resultados que justifiquen el uso in Vivo. No se desconoce el hecho de que los estudio in Vitro no reflejan con exactitud los resultados in Vivo. Sin embargo, con relación a la permeabilidad de la dentina radicular en dientes humanos, Prokopowitsch, Moura e Muench (4) en 1989 verificaron que los métodos de estudio son igualmente validos para evaluar la permeabilidad de la dentina radicular, ya que no existe diferencia estadísticamente significativa entre ambas técnicas.

Por eso, en este estudio fueron empleados 30 primeros premolares superiores humanos hígidos, con formación radicular completa y con raíces separadas.

Fueron utilizados los siguientes láseres, con sus respectivos parámetros sugeridos para el empleo en la clínica: Er:YAG con 2,94 $\mu \mathrm{m}$ - $100 \mathrm{~mJ}, 10 \mathrm{~Hz}, 1 \mathrm{~W}$, con movimiento helicoidal ápico-cervical, $2 \mathrm{~mm}$ por segundo, 4 veces, fibra con 0,375 $\mu \mathrm{m}$ de diámetro, $42 \mathrm{~mJ}$ y para el láser de Nd:YAG con 1,064 $\mu \mathrm{m}-100$ $\mathrm{mJ}, 1,5 \mathrm{~W}, 15 \mathrm{~Hz}$, fibra con $300 \mu \mathrm{m}$, helicoidal ápicocervical, $2 \mathrm{~mm}$ por segundo, 4 veces (20).

En este estudio, para la evaluación de la permeabilidad, usamos el método cuantitativo de penetración del radioisótopo ${ }^{99 \mathrm{~m} T c}$ libre.

Frente a un análisis global, los resultados contenidos en este trabajo, que se refiere a la permeabilidad de la dentina radicular en relación a los grupos experimentales, refuerza la necesidad de una preparación adecuada, que requiere la correcta elección y utilización de una sustancia química auxiliar, con el fin de una desinfección eficaz, incrementado por el uso de los láseres de alta intensidad; ya que el láser de Nd:YAG parece ser el más indicado para mitigar la permeabilidad. Si el objetivo final del uso de la irradiación con láser es la limpieza y la apertura de un mayor número de túbulos dentinarios, el láser de Er:YAG deberá ser de mejor elección. .

Así mismo, proponemos la realización de más estudios para aclarar o buscar una mejor probabilidad en el aumento del poder de desinfección de los canales radiculares en cuanto a la aplicación e irradiación de láser en endodoncia. 


\section{CONCLUSIONES}

Mediante el análisis de los resultados de este estudio y de acuerdo con las metodologías empleadas en la evaluación del índice de permeabilidad, grado de limpieza, calidad de adherencia y contaminación de Enterococcus faecalis, parece razonable concluir que:

La irradiación con láser de Nd:YAG provocó una disminución significativa de permeabilidad dentinaria de las paredes del canal radicular.

El láser de Er:YAG y el control, en relación con la permeabilidad de las paredes del canal radicular, no presentaron diferencia estadísticamente significativa de $5 \%$.

\section{BIBLIOGRAFÍA}

1. Holland GR. The odontoblast process: form and function. J Dent Res. 1985 Apr;64.

2. Camargo $\mathrm{CH}$, Siviero M, Camargo SE, de Oliveira $\mathrm{SH}$, Carvalho CA, Valera MC Topographical, Diametral, and Quantitative Analysis of Dentin Tubules in the Root Canals of Human and Bovine Teeth. JOE 2007 April; 33(4):422-6.

3. Gekelman D, Prokopowitsch I, Eduardo CP. In vitro study of the effects of Nd:YAG laser irradiation on the apical sealing of endodontic fillings performed with and without dentin plugs. J Clin Laser Med Surg. 2002 Jun;20(3):117-21.

4. Prokopowitsch I, de Moura AA, Muench A. "In vitro" analysis of the dentin permeability of the apical third of the root as a function of changes in auxiliary chemicals used in instrumentation. Rev Odontol Univ Sao Paulo. 1989 Apr-Jun;3(2): 345-53. Portuguese.

5. Thaler A, Ebert J, Petschelt A, Pelka M. Influence of tooth age and root section on root dentine dye penetration. Int Endod J. 2008 Dec;41(12): 1115-22.

6. Orosco FA, Bramante CM, Garcia RB, Bernadineli N, Moraes IG. Sealing ability of grar MTA
AngelusTM, CPM TM and MBPc used as apical plugs. J Appl Oral Sci. 2008 Feb;16(1):50-4.

7. Miletic I, Anic I, Pazeij-Ribaric S, Jukic S. Leakage of five root canal sealers. Int Endod J 1999;32: 415-8.

8. Boyd RE. Technetium-99m generators. The available options. Int J Appl Radiat Isot 1982;33: 801-9.

9. Ivanovic V, Pajic M. Quantitative evaluation of permeability of root canalicular system. Stomatol Glas Srb 1990;37(1):11-8.

10. Lever JR. Principles of nuclear medicine. Philadelphia, PA: Saunder, 1995.

11. Araki AT, Ibraki Y, Kawakami T, Lage-Marques JL. Er:YAG laser irradiation of the microbiological apical biofilm.Braz Dent J 2006;17(4):296-9.

12. Bergmans L, Moisiadis P, Teughels W, Van Meerbeek B, Quirynen, Lambrechts P. Bactericidal effects of Nd:YAG laser irradiation on some endodontic pathogens ex vivo. Int Endod J 2006; 39(7):547-57.

13. Gutknecht N, Moritz A, Conrads G, Sievert T, Lampert F. Bactericidal effect of the Nd:YAG laser in vitro root canal. J Clin Laser Med Surg 1996; 14(2):77-80.

14. Harashima T, Takeda FH, Kimura Y, Matsumoto K. Effect of Nd:YAG laser irradiation for removal of intracanal debris and smear layer in extracted human teeth. J Clin Laser Med Surg 1997;15(3): 131-5.

15. Matsuoka E, Kimura Y, Matsumoto K. Studies on the removal of debris near the apical seats by Er:YAG laser and assessment with a fiberscope. J Clin Laser Med Surg. 1998 Oct;16(5):255-61.

16. Stabholz A, Khayat A, Weeks DA, Neev J, Torabinejad M. Scanning electron microscopic study of the apical dentine surfaces lased with ND:YAG laser following apicectomy and retrofill. Int Endod J 1992 Nov;25(6):288-91. 
17. Miserendino LJ, Levy GC, Rizoiu IM. Effects of Nd:YAG laser on the permeability of root canal wall dentin. J Endod 1995 Feb;21(2):83-7.

18. Anic I, Segovic S, Katanec D, Prskalo K, NajzarFleger D. Scanning electron microscopic study of dentin lased with argon, CO2, and Nd:YAG laser. J Endod 1998;24(2):77-81.

19. Lan WH. Temperature elevation the root surface during Nd:YAG laser irradiation in the root canal. J Endod 1999;25(3):155-6.

20. Lin CP, Lee BS, Lin FH, Kok SH, Lan WH. Phase, compositional, and morphological changes of human dentin after Nd:YAG laser treatment. J Endod. 2001;27(6):389-93.

21. Coluzzi DJ. Atlas of laser applications in denstistry /Coluzzi DJ, Convissar RA Quintessence Publishing Co, Inc 2007.

22. Lee BS, Lin CP, Lin FH, Lan WH.Ultrastructural changes of human dentin after irradiation by Nd: YAG laser. Lasers Surg Med 2002;30(3):246-52.

23. Takeda FH, Harashima T, Kimura Y, Matsumoto K. Efficacy of Er: YAG laser irradiation in removing debris and smear layer on root canal walls. J Endod 1998;24(8):548-51.

24. Takeda FH, Harashima T, Kimura Y, Matsumoto K. A comparative study of the removal of smear layer by three endodontic irrigants and two types of laser. Int Endod J. 1999 Jan;32(1):32-9.

25. Biedma BM, Varela Patino P, Park SA, Barciela Castro N, Magan Munoz F, Gonzalez Bahillo JD et al. Comparative study of root canals instrumented manually and mechanically, with and without Er:YAG laser. Photomed Laser Surg 2005;23(5):465-9.

26. Pécora JD, Brugnera-Junior A, Cussioli AL, Zanin $F$, Silva R.Evaluation of dentin root canal permeability after instrumentation and Er:YAG laser application. Lasers Surg Med 2000;26(3): 277-81.

27. Aranha AC, Domingues FB, Franco VO, Gutknecht N, Eduardo CP. Effects of Er:YAG and Nd:YAG lasers on dentin permeability in root surfaces: a preliminary in vitro study. Photomed Laser Surg 2005,23(5):504-8.

\section{CORRESPONDENCIA}

Ricardo Cabrales Salgado.

Facultad de Odontología.

Universidad de Cartagena.

Campus de La Salud, Barrio Zaragocilla.

Cartagena de Indias.

Bolívar. Colombia

Correo electrónico: cabralessrj@yahoo.com 